\title{
Clostridium-difficile-Kolitis
}

\section{Gefahren durch Therapie mit Säureblocker}

Eine Dauertherapie mit Protonenpumpeninhibitoren (PPI) hat zwar keinen Einfluss auf den Blutdruck oder das Auftreten von Pneumonien, dafür erhöht sie das Risiko für Magen-Darm-Infektionen, insbesondere mit Clostridium difficile.

Es gibt kaum eine Nebenwirkung, die nicht schon mit einer PPI-Langzeittherapie in Zusammenhang gebracht wurde. In den meisten Fällen hat sich der Verdacht aber nicht bestätigt, wie Prof. Dr. Jürgen Pohl von der AsklepiosKlinik Altona in Hamburg beim Internisten Update betonte. Als Beispiel nannte er das lange Zeit vermutete Osteoporoserisiko: Für den Anstieg von Oberschenkelhalsfrakturen bei älteren Frauen gebe es "schlechte Evidenz für ein klinisch wahrscheinlich nicht signifikantes Risiko“. Der Gastroenterologe gab auch Entwarnung, was die Resorption von Eisen, Vitamin B 12 und Folsäure betrifft. „PPI haben darauf keinen Einfluss." Auch der Blutdruck werde durch PPI nicht erhöht. Ebenso unbegründet sei die Sorge vor einer verminderten Clopidogrelwirkung.

\section{Keine häufigeren Atemwegsinfekte} Beim Infektionsrisiko sind die Botschaften gemischt. Der Verdacht, PPI könnten Infektionen der oberen Atemwege oder Pneumonien begünstigen, hat sich laut Pohl nicht bestätigt. Dies konnte unter anderem eine kürzlich veröffentlichte Studie zur Lungenentzündung zeigen [1]. Danach haben Patienten, die mit PPI behandelt wurden, zwar häufiger Pneumonien. Die Infektionsrate ist jedoch unabhängig von der säurehemmenden Therapie und schon vorher erhöht.

Bei Magen-Darm-Infektionen hält Pohl einen Zusammenhang mit einer PPI-Dauertherapie dagegen für „wahrscheinlich“. Als "sicher" beurteilt der Gastroenterologe das Risiko für Clostridium-difficileInfektionen. Die Wahrscheinlichkeit einer

Infektion sei unter PPI fast verdoppelt, die Rezidivgefahr um etwa 50\% erhöht.

Als wahrscheinlichste Ursache für die erhöhte Infektionsneigung gilt eine

Protonenpumpenhemmer verringern die Bakterienvielfalt im Darm.

veränderte Kolonisation der Darmschleimhaut. „Das Darmmikrobiom kann je nach Zusammensetzung eine protektive oder permissive Wirkung auf Infektionen haben“, sagte Pohl.

Dass PPI tatsächlich die Zusammensetzung des Mikrobioms verändern, ist in einer Studie gezeigt worden, in der das Bakteriengenom in Stuhlproben komplett sequenziert wurde [2]. Bei den 1600 Nicht-PPI-Anwendern wurde eine höhere Bakterienvielfalt und vor allem ein Übergewicht an Bifidobakterien entdeckt. Bei den 200 PPI-Anwendern fand sich außer einer geringeren Diversität auch ein erheblicher Teil der Flora der Mundschleimhaut sowie ein deutlicher Zuwachs bei Staphylo-, Pneumo- und Enterokokken. „Unter PPI steigt die Durchlässigkeit der Säurebarriere, Bakterien aus dem Mund können besser durchflutschen“, erkärte Pohl.

Erst in diesem Jahr hat eine weitere PPIStudie für Aufregung gesorgt, in der bei älteren Menschen mit PPI-Dauertherapie ein 1,4-fach erhöhtes Risiko für eine Demenzentwicklung festgestellt wurde [3].

Pohl warnte jedoch vor übereilten Schlüssen, schließlich handele es sich um eine Assoziationsstudie mit vielen Fehlermöglichkeiten. Der Gastroenterologe sieht in den Studienergebnissen zunächst einmal einen Weckruf. „Sie sollten uns dazu stimulieren, PPI sinnvoll und indikationsgemäß einzusetzen und den großzügigen Umgang mit PPI zu stoppen.“

\section{Indikationsgerecht behandeln}

Bei der gastroösophagealen Refluxkrankheit sind PPI weiterhin die Therapie der ersten Wahl. Anders als oft gehandhabt sollten die Patienten aber zunächst nur eine einmal tägliche Therapie erhalten. Erst wenn das nicht ausreicht, ist laut Pohl die zweimal tägliche Gabe angezeigt.

Zur Prophylaxe gastroduodenaler Ulzera „sollten“, so die Leitlinie [4], PPI z.B. dann eingesetzt werden, wenn die Patienten eine NSAR-Dauertherapie erhalten und mindestens einen Risikofaktor wie höheres Alter oder Ulkusanamnese aufweisen. Neu ist eine „Soll“-Empfehlung für Patienten, die außer einem NSAR ein Antithrombotikum einnehmen. Kommt zu einer Monotherapie mit einer gerinnungsaktiven Substanz ein Risikofaktor wie höheres Alter hinzu, gilt eine „Kann“-Empfehlung für PPI.

Ärzte müssten aber nicht nur die Indikationen für PPI kennen, betonte Pohl: Sie müssten auch daran denken, PPI abzusetzen, wenn eine Indikation nicht mehr bestehe. "Oft ist eine PPI-Therapie ein Selbstläufer - davor möchte ich warnen." 